\title{
Comprehensive screening for immunodeficiency-associated vaccine-derived poliovirus: an essential oral poliovirus vaccine cessation risk management strategy
}

\section{R. J. DUINTJER TEBBENS* AND K. M. THOMPSON}

Kid Risk, Inc., Orlando, FL, USA

Received 26 March 2016; Final revision 3 June 2016; Accepted 14 September 2016;

first published online 20 October 2016

\section{SUMMARY}

If the world can successfully control all outbreaks of circulating vaccine-derived poliovirus that may occur soon after global oral poliovirus vaccine (OPV) cessation, then immunodeficiencyassociated vaccine-derived polioviruses (iVDPVs) from rare and mostly asymptomatic long-term excretors (defined as $\geqslant 6$ months of excretion) will become the main source of potential poliovirus outbreaks for as long as iVDPV excretion continues. Using existing models of global iVDPV prevalence and global long-term poliovirus risk management, we explore the implications of uncertainties related to iVDPV risks, including the ability to identify asymptomatic iVDPV excretors to treat with polio antiviral drugs (PAVDs) and the transmissibility of iVDPVs. The expected benefits of expanded screening to identify and treat long-term iVDPV excretors with PAVDs range from US $\$ 0.7$ to 1.5 billion with the identification of $25-90 \%$ of asymptomatic long-term iVDPV excretors, respectively. However, these estimates depend strongly on assumptions about the transmissibility of iVDPVs and model inputs affecting the global iVDPV prevalence. For example, the expected benefits may decrease to as low as US\$260 million with the identification of $90 \%$ of asymptomatic iVDPV excretors if iVDPVs behave and transmit like partially reverted viruses instead of fully reverted viruses. Comprehensive screening for iVDPVs will reduce uncertainties and maximize the expected benefits of PAVD use.

Key words: Antiviral drugs, health economics, mathematical modelling, polio, oral poliovirus vaccine.

\section{INTRODUCTION}

Extensive use of the oral poliovirus vaccine (OPV) led to the certified global eradication of wild poliovirus (WPV) of one of three serotypes (i.e. serotype 2) [1], apparent interruption of all serotype 3 WPV (WPV3) transmission [2], and confinement of indigenous serotype 1 WPV (WPV1) transmission to Pakistan, Afghanistan, and Nigeria by 2016 [3]. However, OPV can cause vaccine-associated paralytic poliovirus (VAPP) and

\footnotetext{
* Author for correspondence: Dr R. J. Duintjer Tebbens, Kid Risk, Inc., 10524 Moss Park Rd., Ste. 204-364, Orlando, FL 32832, USA. (Email: rdt@kidrisk.org)
}

evolve to vaccine-derived polioviruses (VDPVs) that can cause outbreaks similar to WPV outbreaks [4]. To end all poliomyelitis disease (i.e. polio), the world stopped all serotype 2-containing OPV use in 2016 and will stop the remaining two serotypes after assurance of global WPV1 and WPV3 eradication [5], leaving the inactivated poliovirus vaccine (IPV) as the only vaccine available to induce individual protection from polio. IPV provides much less protection from participation in faecal-oral transmission than OPV [6-9]. Consequently, in populations with conditions conducive to faecal-oral poliovirus transmission and/ or low routine immunization coverage, population immunity to transmission will decrease rapidly after OPV 
cessation $[10,11]$. OPV intensification prior to OPV cessation, appropriate synchronization and coordination, and aggressive outbreak response can prevent or control circulating VDPV (cVDPV) outbreaks after OPV cessation [12-17], such that the world would subsequently enter an unprecedented era with respect to population immunity to poliovirus transmission. Any outbreaks will become increasingly difficult to contain and OPV use for outbreak response will become increasingly risky due to the possibility of exporting OPV-related viruses to areas outside the target population that can support circulation and evolution of these viruses [13, $14,17]$. IPV likely cannot control a rapidly propagating outbreak in a setting conducive to intense faecal-oral poliovirus transmission [17, 18], and therefore long-term risk management should focus on prevention rather than relying on outbreak response preparedness.

Long-term poliovirus re-introduction risks include breaches in poliovirus containment and introductions of immunodeficiency-associated VDPVs (iVDPV) from rare (i.e. on the order of 100 identified worldwide since OPV use started in the 1960s) long-term poliovirus excretors (defined as $\geqslant 6$ months of excretion) with B-cell-related primary immunodeficiency diseases (PIDs) [19, 20]. Global poliovirus containment efforts and development of non-replicating IPV seed strains aim to minimize the risk of containment breaches [21-23]. Recognizing the iVDPV risks [24], ongoing efforts to develop polio antiviral drugs (PAVDs) led to Phase I and II clinical trials of one compound (pocapavir) and the development of other compounds that may work as a combined therapy to minimize drug resistance [25]. An effective PAVD would offer identified long-term iVDPV excretors protection from developing VAPP by clearing their infection, and this would reduce the risk of iVDPV introductions. However, the identification of iVDPV excretors remains a major challenge because the majority experience no polio symptoms during most or all of their infection [4, 26-29]. Although multiple largely cross-sectional screening studies conducted to date examined $\sim 1000$ PID patients for asymptomatic iVDPV excretion, this represents only a fraction of the estimated 10000 s of PID patients likely alive today [30]. Consequently, most long-term iVDPV excretors identified to date presented with VAPP through the global acute flaccid paralysis surveillance system [4]. Identifying a large fraction of asymptomatic long-term iVDPV excretors will require substantial additional efforts to comprehensively screen PID patients for iVDPVs. Unlike B-cell related primary immunodeficiencies, T-cell immunodeficiency diseases (e.g. HIV) do not appear to lead to long-term iVDPV infections [31, 32].

A global iVDPV prevalence model identified multiple uncertainties that limit our understanding of iVDPV risks [26]. To help inform decisions related to investments in PAVD development and use, we use existing models $[23,26]$ to explore the expected health and economic benefits of efforts to identify asymptomatic long-term iVDPV excretors and the role of key uncertainties related to iVDPV risks.

\section{METHODS}

The discrete-event simulation model (i.e. the DES model) estimates the global prevalence of long-term iVDPV excretors as a function of time after OPV cessation [26]. It probabilistically simulates relevant monthly events over the lifetime of all global PID patients, including (i) birth, (ii) PID onset, diagnosis, and treatment, (iii) OPV infections, recovery, and VAPP, and (iv) death [26]. To characterize the variability between countries, the DES model adopts the stratification of the world into 710 populations with different basic reproductive numbers ( $R_{0}$ values) from an integrated global model of long-term risk management (i.e. the global model) [23]. The DES model uses these $R_{0}$ values as a proxy for hygienic conditions that affect PID survival, with higher $R_{0}$ values implying shorter PID survival. Each stochastic DES model iteration produces a different realization of long-term iVDPV excretors over time in each global model population, which may result in iVDPV introductions into the corresponding populations. The global model integrates multiple components: (i) polio vaccination policy choices, including the global switch from trivalent to bivalent OPV that occurred in late April and early May 2016 and the global cessation of the remaining two OPV serotypes assuming this will occur in 2019, (ii) poliovirus transmission and OPV evolution dynamics [33], (iii) economic inputs related to vaccination costs and the direct and indirect costs associated with polio cases, (iv) stochastic risks after OPV cessation (including iVDPV introductions based on iVDPV prevalence from the DES model), (v) characterization of the global variability in conditions (e.g. $R_{0}$, vaccination coverage) and policies, and (vi) random poliovirus exportations between the 710 populations (structured into epidemiological blocks of 10 subpopulations each and nine larger geographical regions consisting of multiple blocks) 
$[17,23]$. The global model evolves OPV-related viruses from the OPV parent strain (stage 0) given to and excreted by vaccine recipients through a 20 -stage reversion process to fully reverted VDPVs (stage 19) for which we assume an equal $R_{0}$ and paralysis-to-infection ratio (PIR) as typical homotypic WPVs [33]. The global model also tracks all PID patients from the DES model to account for the possibility that monovalent OPV (mOPV) use for outbreak response could generate new long-term iVDPV infections after OPV cessation. Introductions may or may not lead to sufficient initial transmissions to generate an outbreak, which the global model accounts for by randomly determining whether an introduction starts population-wide transmission (i.e. defined as an effective introduction) based on population immunity to transmission and the $R_{0}$ of the introduced virus. Thus, populations at highest risk of outbreaks given an iVDPV introduction coincide with those in which PID survival remains the shortest.

An economic analysis used the global model to estimate the expected incremental net benefits of major long-term risk management policies involving OPV cessation compared to continued OPV use with or without continued supplemental immunization activities (SIAs) [23]. The base case assumed minimum global IPV use of at least one routine immunization dose for 5 years after cessation of the last OPV serotype in 2019 (policy abbreviation IPV5). The policy specifically assumes that populations that already used IPV in 2013 and upper middle-income populations (which we assumed switch to a three-dose IPV schedule at cessation of the last OPV serotype) continue to use threedose IPV routine immunization schedules until the end of the analytical time horizon that extends for 40 years after the most recent Global Polio Eradication Initiate Strategic Plan took effect (i.e. $T_{\text {end }}=2053$ [5]). In addition to IPV use and sufficient OPV intensification before OPV cessation of each serotype to prevent cVDPV outbreaks, the global model base case assumed aggressive outbreak response and relatively low risks of non-iVDPV long-term poliovirus re-introductions. Recognizing the risks that even the attenuated strains in OPV could establish circulation when population immunity to poliovirus transmission becomes very low and that widespread OPV use could start new longterm iVDPV infections, the outbreak response strategy assumed mOPV use for only the first 5 years after homotypic OPV cessation and IPV thereafter. In the rare event of uncontrolled outbreaks after OPV cessation, rather than continuing reactive outbreak response SIAs with IPV, the global model assumed that reaching an arbitrary threshold of 50000 polio cases globally would lead to resumed OPV use in all countries that used OPV as of 2013 and that from then on the world would incur the expected costs and cases associated with OPV use in routine immunization and any SIAs. We refer to stochastic iterations in which this occurs as OPV restart iterations [23]. Variations around the base case without PAVD use included two scenarios of PAVD use starting on 1 January 2017. The first variation (PAVD $40 \%$ scenario) assumed $40 \%$ PAVD effectiveness and that $50 \%$ of long-term iVDPV excretors who presented clinically with VAPP receive PAVDs. The second variation (PAVD90\% scenario) assumed high PAVD effectiveness of $90 \%$ and that $90 \%$ of all long-term iVDPV excretors (including those with no paralytic symptoms) receive PAVDs.

This study first performs a probabilistic uncertainty and sensitivity analysis of the DES model (see Supplementary material section $\mathrm{S} 1$ ). We then consider in more detail the role of screening for asymptomatic long-term iVDPV excretors, assuming the global model base case for all other assumptions and a stratified set of 120 iterations (see Supplementary material section S2). Assuming a 90\% effective combined PAVD therapy and that the existing acute flaccid paralysis surveillance system would allow PAVD administration to $90 \%$ of long-term iVDPV excretors at VAPP onset, we vary the fraction of asymptomatic long-term iVDPV excretors identified through the screening programme and receiving PAVDs [i.e. the identification fraction (IF)] between $25 \%$ and $90 \%$. For each IF, we report global outcomes related to iVDPV risks and the health-economic benefits of the PAVD use. We do not include the costs of the iVDPV screening efforts. Consistent with the global model, we expressed monetary amounts in 2013 US dollars (\$) and used a 3\% discount rate.

We also consider the uncertainty about the transmissibility and neurovirulence of iVDPVs. While our prior work consistently assumed the same $R_{0}$ and PIR for cVDPVs and iVDPVs as homotypic WPVs because no direct evidence exists to support lower values for VDPVs [20, 26, 33, 34], some possibility exists that iVDPVs could behave differently due to antigenic adaptations that may occur during prolonged replication in limited intestinal sites of a single host. Although the absence of any known outbreak despite over 70 known long-term iVDPV excretors to date reflects high surrounding population immunity to transmission through vaccination, it could also partly reflect lower $R_{0}$ values for iVDPVs 
Table 1. The contribution of key inputs to the uncertainty in the DES model [26], ranked by absolute values of the rank correlation between each input and the time until the last iVDPV excretor anywhere in the world stops excreting in the DES model

\begin{tabular}{|c|c|c|c|c|c|}
\hline \multirow[b]{2}{*}{ DES model input } & \multicolumn{5}{|c|}{$\begin{array}{l}\text { Rank correlation between given model input and time when last iVDPV } \\
\text { excretor stops excreting in }\end{array}$} \\
\hline & World & $\begin{array}{l}\text { Low-income } \\
\text { countries }\end{array}$ & $\begin{array}{l}\text { Lower } \\
\text { middle-income } \\
\text { countries }\end{array}$ & $\begin{array}{l}\text { Upper } \\
\text { middle-income } \\
\text { countries }\end{array}$ & $\begin{array}{l}\text { High-income } \\
\text { countries }\end{array}$ \\
\hline Average duration of iVDPV infection (years) & $0 \cdot 49$ & $0 \cdot 10$ & $0 \cdot 12$ & $0 \cdot 11$ & $0 \cdot 56$ \\
\hline Relative monthly death rate $v s$. baseline & $-0 \cdot 48$ & $-0 \cdot 66$ & $-0 \cdot 65$ & -0.68 & $-0 \cdot 35$ \\
\hline Potential long-term excretion probability & $0 \cdot 39$ & $0 \cdot 45$ & $0 \cdot 46$ & $0 \cdot 43$ & $0 \cdot 41$ \\
\hline PID predisposition probability per birth & $0 \cdot 18$ & $0 \cdot 16$ & $0 \cdot 15$ & $0 \cdot 17$ & $0 \cdot 18$ \\
\hline Increase in all OPV exposure rates & $0 \cdot 07$ & $0 \cdot 07$ & $0 \cdot 10$ & $0 \cdot 07$ & $0 \cdot 07$ \\
\hline $\begin{array}{l}\text { Relative probability of long-term OPV } \\
\text { infection if treated } v s \text {. not treated }\end{array}$ & $-0 \cdot 06$ & $-0 \cdot 04$ & $-0 \cdot 05$ & $-0 \cdot 07$ & $-0 \cdot 04$ \\
\hline Monthly PID onset probability & $0 \cdot 00$ & $0 \cdot 07$ & $0 \cdot 02$ & $-0 \cdot 01$ & $0 \cdot 02$ \\
\hline
\end{tabular}

DES, Discrete-event simulation; iVDPV, immunodeficiency-associated vaccine-derived poliovirus; OPV, oral poliovirus vaccine; PID, primary immunodeficiency disease.

than WPVs. To explore this possibility, we run the IPV5 policy both without PAVDs and with an IF of $90 \%$, assuming that iVDPV introductions into the subpopulation that the excretor resides in occur at partially reverted stage 10 instead of the fully reverted VDPV stage (stage 19). This implies that any iVDPV introductions start with an $R_{0}$ and $\log$ PIR about halfway between those of the OPV parent strain and homotypic WPVs [33]. The lower $R_{0}$ decreases the chances that the introduction leads to an outbreak, but it does not preclude further evolution of these viruses as they circulate until they reach the fully reverted stage.

\section{RESULTS}

Table 1 shows the relative importance of uncertain inputs based on 1000 DES model iterations, with rank correlations closer to $1(-1)$ indicating a stronger increasing (decreasing) effect of an input on the time until the last long-term iVDPV excretor stops excreting (Supplementary material section S1). Globally, the last excretor stops excreting in early 2028 on average (s.D. = $6 \cdot 0$ years) in the DES model with all uncertain inputs at their base case values. With the input uncertainty included, this average time increases by 4.4 years to mid-2032 (s.D. $=9 \cdot 8$ years). Uncertainty about the average duration of long-term iVDPV infections contributes most to the uncertainty about the time until the last iVDPV excretor in the world stops excreting. However, this results mainly from the possibility that a chronic excretor in a high-income country survives and remains infected for decades after OPV cessation, which represents a lesser concern globally because of the expected continued high IPV-induced population immunity to transmission in those countries. In lowand middle-income countries, in which population immunity to transmission will decrease to unprecedented low levels after OPV cessation [23], the uncertainty about the time until the last iVDPV excretor stops excreting depends most on the uncertainty about PID survival, followed by the potential long-term excretion probability and incidence of PID-predisposed births. Other inputs in Table 1 matter less because they primarily affect how many long-term iVDPV excretors exist at the time of OPV cessation with no or little effect on how long they subsequently remain infected.

Table 2 shows the effect of efforts to identify and treat asymptomatic long-term iVDPV excretors with PAVDs on various global model outcomes and reveals a consistent reduction in undesirable outcomes with increasing IF. Specifically, a higher IF means that more long-term iVDPV excretors clear their infection after receiving PAVDs, which reduces the expected number of iVDPV introductions, including from any long-term iVDPV excretors affected by mOPV used for outbreak response. This reduces the probability of outbreaks, including uncontrolled outbreaks leading to OPV restarts (see Supplementary material section S2 for characteristics of OPV restart iterations). Increased PAVD use also reduces the number of doses required for outbreak response SIAs, the probability of unmet stockpile vaccine needs and associated 
Table 2. Global model results for different assumptions about the IF, based on a stratified set of 120 stochastic iterations

\begin{tabular}{|c|c|c|c|c|c|}
\hline Global model outcome & $\begin{array}{l}\text { Base case } \\
\text { (i.e. no } \\
\text { PAVDs) }\end{array}$ & $\begin{array}{l}\mathrm{IF}=25 \% \\
\text { (decrease } v s . \\
\text { base case) }\end{array}$ & $\begin{array}{l}\text { IF }=50 \% \\
\text { (decrease } v s . \\
\text { base case) }\end{array}$ & $\begin{array}{l}\text { IF }=75 \% \\
\text { (decrease } v s . \\
\text { base case) }\end{array}$ & $\begin{array}{l}\mathrm{IF}=90 \% \\
\text { (decrease } v s . \\
\text { base case) }\end{array}$ \\
\hline \multicolumn{6}{|l|}{ Average* number of effective iVDPV introductions } \\
\hline From iVDPV excretors infected by mOPV SIAs & $0 \cdot 18$ & $0 \cdot 018(0 \cdot 16)$ & $0 \cdot 0030(0 \cdot 17)$ & $0(0 \cdot 18)$ & $0(0 \cdot 18)$ \\
\hline From all iVDPV excretors & 16 & $12(4 \cdot 7)$ & $9 \cdot 2(7 \cdot 3)$ & $6 \cdot 1(10)$ & $5 \cdot 0(12)$ \\
\hline Probability of at least one outbreak & $0 \cdot 95$ & $0 \cdot 91(0 \cdot 049)$ & $0 \cdot 81(0 \cdot 14)$ & $0 \cdot 66(0 \cdot 30)$ & $0 \cdot 56(0 \cdot 40)$ \\
\hline OPV restart probability & $0 \cdot 057$ & $0.036(0.021)$ & $0.032(0 \cdot 025)$ & $0 \cdot 019(0 \cdot 038)$ & $0 \cdot 013(0 \cdot 044)$ \\
\hline \multicolumn{6}{|l|}{$\begin{array}{l}\text { Average number of outbreak response SIA doses } \\
\text { used (millions) }\end{array}$} \\
\hline mOPV & 150 & $110(38)$ & $92(58)$ & $47(100)$ & $27(120)$ \\
\hline IPV & 780 & $440(340)$ & $410(370)$ & $180(600)$ & $98(680)$ \\
\hline Both & 930 & $550(380)$ & $500(430)$ & $230(700)$ & $120(810)$ \\
\hline \multicolumn{6}{|l|}{ Outbreak response SIA costs ( $\$$ millions) } \\
\hline mOPV & 100 & $75(26)$ & $61(41)$ & $32(70)$ & $18(83)$ \\
\hline IPV & 510 & $290(220)$ & $270(240)$ & $123(380)$ & $70(440)$ \\
\hline Both & 610 & $360(250)$ & $330(280)$ & $150(450)$ & $88(520)$ \\
\hline Average number of new long-term iVDPV infections & $1 \cdot 2$ & $0 \cdot 91(0 \cdot 31)$ & $0.69(0.52)$ & $0 \cdot 45(0 \cdot 77)$ & $0 \cdot 26(0 \cdot 95)$ \\
\hline \multicolumn{6}{|l|}{ Average number of polio cases from 2016 on (thousands) } \\
\hline 57 iterations with OPV restart without SIAs $\uparrow$ & 1100 & $660(400)$ & $600(460)$ & $340(730)$ & $230(840)$ \\
\hline 57 iterations with OPV restart with SIAs $\dagger$ & 200 & $130(71)$ & $120(77)$ & $68(130)$ & $47(150)$ \\
\hline 63 iterations without OPV restart & $0 \cdot 57$ & $0 \cdot 50(0 \cdot 071)$ & $0 \cdot 48(0 \cdot 089)$ & $0 \cdot 41(0 \cdot 16)$ & $0 \cdot 38(0 \cdot 19)$ \\
\hline All iterations, assuming OPV restarts without SIAs & 61 & $38(23)$ & $35(26)$ & $20(41)$ & $13(48)$ \\
\hline All iterations, assuming OPV restarts with SIAs & 12 & $7 \cdot 8(4 \cdot 1)$ & $7 \cdot 5(4 \cdot 5)$ & $4 \cdot 3(7 \cdot 6)$ & $3 \cdot 1(8 \cdot 9)$ \\
\hline \multicolumn{6}{|l|}{ Incremental net benefits ( $\$$ billions) of IPV5 compared to: } \\
\hline RC no SIAs, assuming OPV restarts without SIAs & 12 & $13(-0 \cdot 72)$ & $13(-0 \cdot 81)$ & $14(-1 \cdot 3)$ & $14(-1 \cdot 5)$ \\
\hline RC with SIAs, assuming OPV restarts with SIAs & 16 & $16(-0 \cdot 79)$ & $16(-0 \cdot 79)$ & $17(-1 \cdot 3)$ & $17(-1 \cdot 5)$ \\
\hline
\end{tabular}

IF, Identification fraction; IPV, inactivated poliovirus vaccine; IPV5, baseline global policy of at least 5 years of IPV years after global OPV cessation of the last serotype iVDPV, immunodeficiency-associated vaccine-derived poliovirus; mOPV, monovalent OPV; OPV, oral poliovirus vaccine; PAVD, polio antiviral drug; RC, reference case;

SIA, supplemental immunization activity; \$, year 2013 United States dollars.

* All averages represent weighted averages for the stratified set of iterations (see Supplementary material section S2).

$\dagger$ Includes all OPV restart iterations from the stratified set for all columns, such that with PAVDs the averages includes iterations both with and without restarts. The averages

depend on whether we assume that the restart would involve resumption of OPV SIAs in addition to routine immunization. 


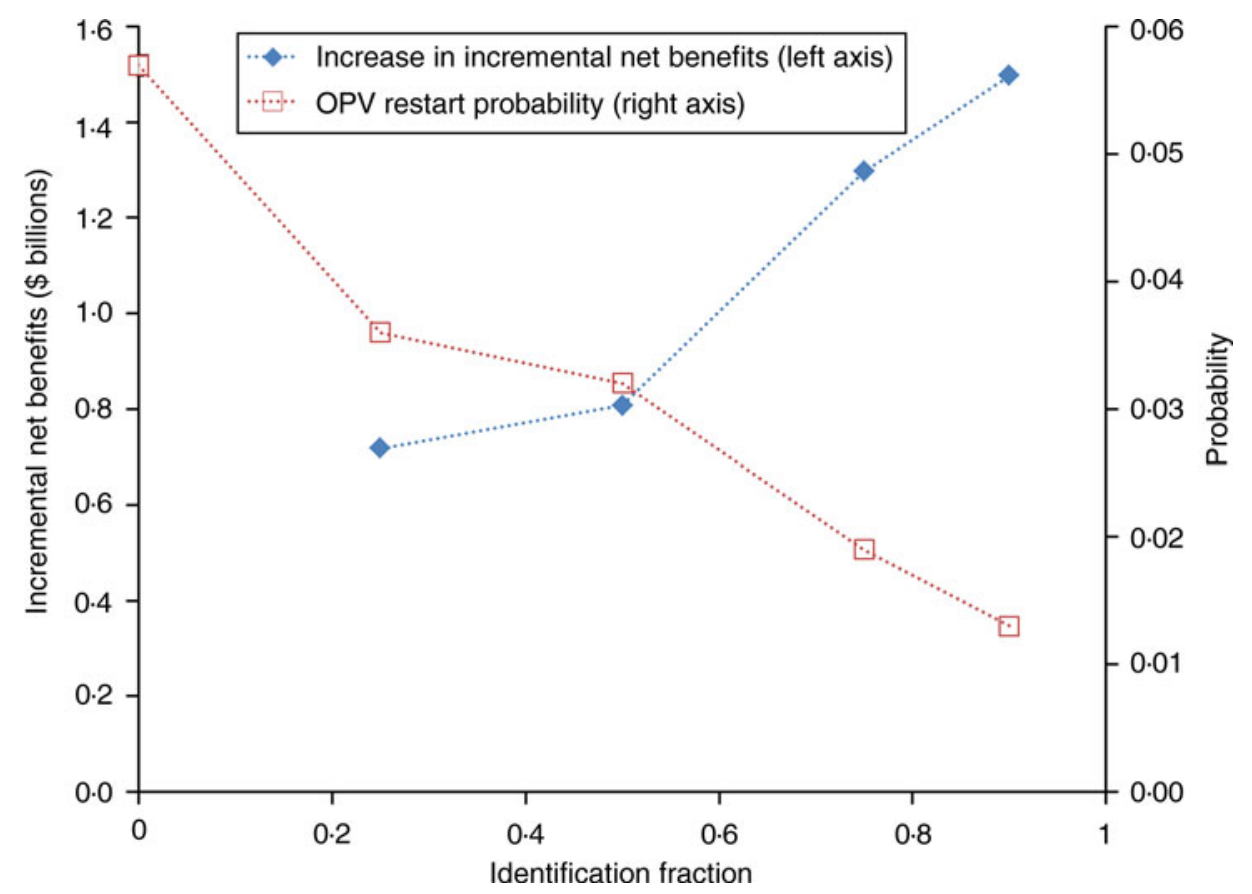

Fig. 1. Relationship between identification fraction and oral poliovirus vaccine (OPV) restart probability (based on 57 OPV restart iterations) and the resulting increase in the incremental net benefits in year 2013 United States dollars (\$) of the baseline policy of at least 5 years of inactivated poliovirus vaccine use after global cessation of the last OPV serotype compared to the reference case without supplemental immunization activities (base case OPV restart probability shown in figure as identification fraction of 0 ).

additional (uncontrolled) outbreaks [17]. Fewer outbreak response SIAs lowers the costs for outbreak response and results in expected financial savings between \$250-520 million (undiscounted) for IFs from $0 \cdot 25$ to $0 \cdot 90$, mainly by avoiding perhaps unrealistically large numbers of IPV outbreak response SIAs that precede OPV restarts. The reduction in outbreak response SIAs with mOPV also reduces the number of PID patients infected by mOPV that develop new longterm iVDPV infections.

Overall, by preventing outbreaks, PAVD use may avoid many OPV restarts that occur for the base case and the associated large numbers of polio cases [23]. While these iterations represent only $5 \cdot 7 \%$ of all expected realizations (Supplementary material section S2), we observed reductions in average polio cases after 2016 even for the much more common iterations without OPV restarts. The percent reductions in these global model results remain somewhat limited because $\sim 350$ VAPP cases occur between 2016 and cessation of the last OPV serotype in 2019 regardless of PAVD use [23], but the absolute reductions in expected polio cases in iterations without OPV restart remain significant (i.e. 71 and 190 for IF $=25 \%$ and $90 \%$, respective1y). The decrease in polio cases and costs with PAVD use increases the incremental net benefits of OPV cessation compared to continued OPV use. For example, the expected incremental net benefits of IPV5 compared to the reference case of continued OPV use without SIAs increase from $\$ 12$ billion without PAVDs to $\$ 13$ billion with IF $=25-50 \%$ and to $\$ 14$ billion with $\mathrm{IF}=75-90 \%$.

Figure 1 highlights the effect of the IF on the OPV restart probability and resulting increase in the incremental net benefits of IPV 5 compared to the reference case without SIAs. These results do not include PAVD development or iVDPV screening costs, but they provide bounding estimates of the potential long-term benefits of making such investments. We estimate potential benefits of PAVDs ranging from $\$ 700$ million (IF $=25 \%$ ) to $\$ 1.5$ billion (IF $=90 \%$ ). However, these estimates depend on numerous assumptions in the model about outbreak consequences (e.g. outbreak response strategy and the frequency of poliovirus exportations to other blocks) and the relative importance of iVDPV risks compared to other risks (e.g. frequency of containment releases, pre-cessation OPV intensification).

Table 3 explores the effect of the uncertainty about the transmissibility of iVDPVs when introduced into the subpopulation in which the excretor resides. All 
Table 3. Global model results for different assumptions about the reversion stage of iVDPVs at the time of introduction into the subpopulation that the excretor resides in, based on a stratified set of 120 stochastic iterations

\begin{tabular}{|c|c|c|c|c|}
\hline \multirow[b]{2}{*}{ Global model outcome } & \multicolumn{2}{|c|}{$\begin{array}{l}\text { Base case (no PAVDs), with iVDPV } \\
\text { introductions in given reversion stage }\end{array}$} & \multicolumn{2}{|c|}{$\begin{array}{l}\text { IF }=90 \%, \text { iVDPV introduction in given } \\
\text { reversion stage (decrease } v s . \text { base case with } \\
\text { same reversion stage assumption) }\end{array}$} \\
\hline & 19 (fully reverted) & 10 (partially reverted) & 19 (fully reverted) & 10 (partially reverted) \\
\hline \multicolumn{5}{|l|}{ Average* number of effective iVDPV introductions } \\
\hline From iVDPV excretors infected by mOPV & $0 \cdot 18$ & $0 \cdot 031$ & $0(0 \cdot 18)$ & $0(0 \cdot 031)$ \\
\hline From all iVDPV excretors & 16 & $17 \dagger$ & $5 \cdot 0(12)$ & $5 \cdot 0 \ddagger(12)$ \\
\hline Probability of at least one outbreak & $0 \cdot 95$ & $0 \cdot 17$ & $0 \cdot 56(0 \cdot 40)$ & $0.071(0 \cdot 095)$ \\
\hline OPV restart probability & $0 \cdot 057$ & $0 \cdot 014$ & $0 \cdot 013(0 \cdot 044)$ & $0 \cdot 007(0 \cdot 007)$ \\
\hline \multicolumn{5}{|c|}{ Average number of outbreak response SIA doses used (millions) } \\
\hline mOPV & 150 & 27 & $27(120)$ & $0 \cdot 86(26)$ \\
\hline IPV & 780 & 170 & $100(680)$ & $52(120)$ \\
\hline Both & 930 & 200 & $120(810)$ & $53(140)$ \\
\hline \multicolumn{5}{|l|}{ Outbreak response SIA costs (\$ millions) } \\
\hline $\mathrm{mOPV}$ & 100 & 17 & $18(83)$ & $0 \cdot 53(16)$ \\
\hline IPV & 510 & 113 & $70(440)$ & $41(72)$ \\
\hline Both & 610 & 130 & $88(520)$ & $41(89)$ \\
\hline Average number of new long-term iVDPV infections & $1 \cdot 2$ & $0 \cdot 20$ & $0 \cdot 26(0 \cdot 95)$ & $0(0 \cdot 20)$ \\
\hline \multicolumn{5}{|l|}{ Average number of polio cases from 2016 on (thousands) } \\
\hline 57 iterations with OPV restart without SIAs: & 1100 & 240 & $230(840)$ & $110(130)$ \\
\hline 57 iterations with OPV restart with SIAst & 200 & 49 & $47(150)$ & $30(19)$ \\
\hline 63 iterations without OPV restart & $0 \cdot 57$ & $0 \cdot 47$ & $0 \cdot 38(0 \cdot 19)$ & $0 \cdot 37(0 \cdot 092)$ \\
\hline All iterations, assuming OPV restarts without SIAs & 61 & 14 & $13(48)$ & $6 \cdot 5(7 \cdot 7)$ \\
\hline All iterations, assuming OPV restarts with SIAs & 12 & $3 \cdot 2$ & $3 \cdot 1(8 \cdot 9)$ & $2 \cdot 1(1 \cdot 2)$ \\
\hline \multicolumn{5}{|l|}{ Incremental net benefits of IPV5 compared to ( $\$$ billions) } \\
\hline RC no SIAs, assuming OPV restarts without SIAs & 12 & 14 & $14(-1 \cdot 5)$ & $14(-0 \cdot 26)$ \\
\hline RC with SIAs, assuming OPV restarts with SIAs & 16 & 17 & $17(-1 \cdot 5)$ & $17(-0 \cdot 26)$ \\
\hline
\end{tabular}

IF, Identification fraction; IPV, inactivated poliovirus vaccine; IPV5, baseline global policy of at least 5 years of IPV years after global OPV cessation of the last serotype; iVDPV, immunodeficiency-associated vaccine-derived poliovirus; mOPV, monovalent OPV; OPV, oral poliovirus vaccine; PAVD, polio antiviral drug; RC, reference case; SIA, supplemental immunization activity; \$, year 2013 United States dollars.

* All averages represent weighted averages for the stratified set of iterations (see Supplementary material section S2).

$\dagger$ The number of effective iVDPV introductions does not decrease for partially-reverted iVDPV introductions because the lack of substantial outbreaks associated with earlier iVDPVs introductions from the same or other long-term iVDPV excretors in the same population allows population immunity to continue to drop, which increases the probability that subsequent introductions become effective.

\$ Includes all OPV restart iterations from the stratified set for all columns, such that with PAVDs and/or partially reverted iVDPV introductions the averages includes iterations both with and without restarts. The averages depend on whether we assume that the restart would involve resumption of OPV SIAs in addition to routine immunization. 
prior results assumed iVDPVs behave like homotypic WPVs and cVDPVs when introduced into a subpopulation. If we assume instead that iVDPV strains did not acquire the same inherent transmissibility as homotypic WPVs but represent only partially-reverted VDPV virus (i.e. introducing them in reversion stage 10 instead of 19), then this markedly reduces iVDPV risks. First, the probability decreases that a contact between an iVDPV excretor and another individual in its subpopulation leads to an effective introduction that can start to transmit at the population level. Second, even effective introductions may die out before substantial circulation due to seasonality or other outbreak kinetics [14], or may stop more easily after an aggressive outbreak response. Consequently, introducing iVDPVs as partially reverted viruses yields large reductions in the probability of outbreaks and OPV restarts, expected outbreak response vaccine needs, and expected polio cases, which translates into higher incremental net benefits for both the base case without PAVDs and for the option with PAVDs. The benefits of PAVD use with IF $=90 \%$ decrease from $\$ 1.5$ billion with fully reverted iVDPV introductions to \$260 million with partially reverted iVDPV introductions.

\section{DISCUSSION}

This study explored several important uncertainties related to iVDPV risks after OPV cessation and the potential use of PAVDs to mitigate these risks. We find very high potential benefits of identifying asymptomatic long-term iVDPV excretors and treating them with effective PAVDs that may become available soon in the form of a combination therapy with at least two synergistic compounds [25]. These benefits range from an expected $\$ 700$ million for an IF of $25 \%$ to $\$ 1.5$ billion for an IF of $90 \%$ if iVDPVs possess the same transmission potential as WPVs and cVPDVs. The upper end represents a higher estimate than our previously reported estimate of $\$ 500$ million based on an initial set of 100 global model iterations that included only one OPV restart associated with iVDPV outbreaks (i.e. probability of $1 \%$ ) [23]. The estimate in this study draws from a much larger set of 1000 global model iterations, including 52 of $57 \mathrm{OPV}$ restarts associated with iVDPV excretors (i.e. probability of $5.2 \%$, see Supplementary material section S2).

The true benefits of PAVDs will depend on the actual realization of the uncertain future, with the observation of iVDPV2 outbreaks (or lack thereof) following the trivalent to bivalent OPV switch within the next few years already potentially providing some answers for serotype 2 that may inform decisions related to serotypes 1 and 3. Better understanding of the true transmissibility of iVDPVs would reduce uncertainty about the expected benefits of PAVD use. Resolving uncertainty about iVDPV prevalence (e.g. through better estimates of PID survival in developing countries) will affect the time until the last iVDPV excretor stops excreting in each income level, which will influence the potential number of cases prevented due to iVDPV outbreaks through the use of PAVDs. Other limitations and uncertainties from the global model [23] and the underlying poliovirus transmission and OPV evolution model [33] carry over to this analysis. Limitations and uncertainties that may particularly affect PAVD benefits include the kinetics of outbreaks (both during the initial stages following a point introduction [14] and the frequency of longrange poliovirus exportations [23]), the impact of IPV-alone on population immunity to transmission in developing countries [10,35], the relationship between population immunity to transmission and the probability that an IVDPV or other introduction establishes transmission, and efforts to manage cVDPV [10, 12 14] and laboratory containment risks [20, 36, 37]. The actual duration of mOPV use for outbreak response and the availability of any new poliovirus vaccines with lower risks than OPV may further affect the risk of uncontrolled outbreaks due to iVDPVs and the benefits of PAVDs [38].

While the inherent transmissibility of iVDPVs remains very challenging to study [26], the assumption of equal transmissibility as homotypic WPV should remain the default assumption for the purposes of risk management unless evidence proves otherwise. All existing in vitro and animal data suggest no difference in phenotypic properties [4, 39], and widespread live poliovirus exposure in developing countries and/ or IPV use in developed countries to date limit the epidemiological observability of iVDPV transmissibility. Further data collection of PID patients in developing countries and further screening for iVDPVs could reduce the uncertainty about: (i) PID survival rates, (ii) the probability that a PID develops a long-term infection, and (iii) the average duration of long-term iVDPV infections. The detection of a relatively high number of long-term iVDPV excretors from countries with high rates of consanguinity $[4,30,40]$ may indicate that these countries experience a higher incidence of genetic predisposition to PIDs per birth, a higher risk of long-term iVDPV infections given OPV 
exposure for PIDs associated with consanguinity than other PIDs, higher OPV exposure rates, better PID survival, or some combination of these factors. Teasing out the possible explanations will require additional studies. Expanding iVDPV screening and continued development of PAVD combination therapies will take time and require resources. Further modeling may help characterize the costs and explore the impacts of different times to implement extensive PAVD use.

Despite the limitations and uncertainties, this study supports substantial investments in PAVDs and expanded screening and treatment of asymptomatic long-term iVDPV excretors as a key long-term polio risk management strategy. A growing network of physicians treating patients with PIDs [30, 41] may offer an opportunity to encourage PID patient screening in the existing global acute flaccid paralysis surveillance system by including stool sample results from all newly identified PID patients in global poliovirus surveillance reporting. As long as OPV use continues, a comprehensive screening system would require continued periodic screening of PID patients for poliovirus and regular follow-up of excretors to track persistent infections. After successful OPV cessation, no live poliovirus circulation should occur, such that screening for poliovirus upon diagnosis of every new PID patient should suffice to identify any iVDPV infections. However, the reality of PID underdiagnosis and the diagnostic delay [42-44] represent important obstacles we must address to achieve high sensitivity of iVDPV screening. Given our findings of risks of problematic outbreaks associated with prolonged iVDPV excretors who do not stop excretion within two years of OPV cessation in countries with high poliovirus transmissibility, we emphasize the urgency of efforts to develop effective PAVDs and expand screening efforts for asymptomatic long-term iVDPV excretors.

\section{SUPPLEMENTARY MATERIAL}

For supplementary material accompanying this paper visit http://dx.doi.org/10.1017/S0950268816002302.

\section{ACKNOWLEDGEMENTS}

This work was supported by Bill and Melinda Gates Foundation [OPP1129391]. The contents of this manuscript are solely the responsibility of the authors.

\section{DECLARATION OF INTEREST}

None.

\section{REFERENCES}

1. Polio Global Eradication Initiative. Global eradication of wild poliovirus type 2 declared (http://www.polioeradication.org/mediaroom/newsstories/Global-eradicationof-wild-poliovirus-type-2-declared/tabid/526/news/1289/ Default.aspx). Accessed 30 November 2015.

2. Kew OM, et al. Possible eradication of wild poliovirus type 3-worldwide, 2012. Morbidity and Mortality Weekly Report 2014; 63: 1031-1033.

3. World Health Organization. Polio this week as of 10 August 2016. (http://www.polioeradication.org/Dataand monitoring/Poliothisweek.aspx). Accessed 14 August 2016.

4. Burns C, et al. Vaccine-derived polioviruses. Journal of Infectious Diseases 2014; 210: S283-S293.

5. World Health Organization. Global Polio Eradication Initiative: polio eradication and endgame strategic plan (2013-2018). Geneva, 2013. Report No. WHO/ POLIO/13.02.

6. Onorato IM, et al. Mucosal immunity induced by enhanced-potency inactivated and oral polio vaccines. Journal of Infectious Diseases 1991; 163: 1-6.

7. Hird TR, Grassly NC. Systematic review of mucosal immunity induced by oral and inactivated poliovirus vaccines against virus shedding following oral poliovirus challenge. PLoS Pathogens 2012; 8: e1002599.

8. Duintjer Tebbens RJ, et al. Expert review on poliovirus immunity and transmission. Risk Analysis 2013; 33: 544-605.

9. Duintjer Tebbens RJ, et al. Review and assessment of poliovirus immunity and transmission: Synthesis of knowledge gaps and identification of research needs. Risk Analysis 2013; 33: 606-646.

10. Duintjer Tebbens RJ, Thompson KM. Modeling the potential role of inactivated poliovirus vaccine to manage the risks of oral poliovirus vaccine cessation. Journal of Infectious Diseases 2014; 210: S485-S497.

11. Kalkowska DA, et al. Modeling options to manage type 1 wild poliovirus imported into Israel in 2013. Journal of Infectious Diseases 2015; 211: 1800-1812.

12. Thompson KM, Duintjer Tebbens RJ. Modeling the dynamics of oral poliovirus vaccine cessation. Journal of Infectious Diseases 2014; 210: S475-S484.

13. Duintjer Tebbens RJ, Hampton LM, Thompson KM. Implementation of coordinated global serotype 2 oral poliovirus vaccine cessation: Risks of potential nonsynchronous cessation. BMC Infectious Diseases 2016; 16: 237 .

14. Duintjer Tebbens RJ, Hampton LM, Thompson KM. Implementation of coordinated global serotype 2 oral poliovirus vaccine cessation: risks of inadvertent trivalent oral poliovirus vaccine use. BMC Infectious Diseases 2016; 16: 231. 
15. Global Polio Eradication Initiative. Operational framework for monovalent oral poliovirus type 2 (mOPV2) deployment and replenishment (during the endgame period) (http://www.polioeradication.org/Portals/0/Document/ Resources/PostEradication/mOPV2_Operational_ Framework.pdf). Accessed 25 September 2015.

16. Global Polio Eradication Initiative. World ready for OPV2 cessation (http://www.polioeradication.org/mediaroom/newsstories/World-ready-for-OPV2-cessation-/ tabid/526/news/1181/Default.aspx?popUp=true). Accessed 28 January 2015.

17. Duintjer Tebbens RJ, et al. Characterization of outbreak response strategies and potential vaccine stockpile needs for the polio endgame. BMC Infectious Diseases 2016; 16: 137 .

18. Anis E, et al. Insidious reintroduction of wild poliovirus into Israel, 2013. Eurosurveillance 2013; 18: pii $=20586$.

19. Aylward RB, Cochi SL. Framework for evaluating the risks of paralytic poliomyelitis after global interruption of wild poliovirus transmission. Bulletin of the World Health Organization 2004; 82: 40-46.

20. Duintjer Tebbens RJ, et al. Risks of paralytic disease due to wild or vaccine-derived poliovirus after eradication. Risk Analysis 2006; 26: 1471-1505.

21. Okayasu H, et al. Affordable inactivated poliovirus vaccine: strategies and progress. Journal of Infectious Diseases 2014; 210 (Suppl. 1): S459-S464.

22. World Health Organization. GAP III: WHO Global Action Plan to minimize poliovirus facility-associated risk after type-specific eradication of wild polioviruses and sequential cessation of oral polio vaccine use (http://www.polioeradication.org/Portals/0/Document/ Resources/PostEradication/GAPIII_2014.pdf). Accessed 9 November 2015.

23. Duintjer Tebbens RJ, et al. An economic analysis of poliovirus risk management policy options for 20132052. BMC Infectious Diseases 2015; 15: 389.

24. National Research Council. Exploring the role of antiviral drugs in the eradication of polio: workshop report. Washington, D.C.: National Academy Press, 2006.

25. McKinlay MA, et al. Progress in the development of poliovirus antiviral agents and their essential role in reducing risks that threaten eradication. Journal of Infectious Diseases 2014; 210: S447-453.

26. Duintjer Tebbens RJ, Pallansch MA, Thompson KM. Modeling the prevalence of immunodeficiencyassociated long-term vaccine-derived poliovirus excretors and the potential benefits of antiviral drugs. $B M C$ Infectious Diseases 2015; 15: 379.

27. Dunn G, et al. Twenty-eight years of poliovirus replication in an immunodeficient individual: impact on the Global Polio Eradication Initiative. PLoS Pathogens 2015; 11: e1005114.

28. Bellmunt A, et al. Evolution of poliovirus type I during 5.5 years of prolonged enteral replication in an immunodeficient patient. Virology 1999; 265: 178-184.
29. DeVries AS, et al. Vaccine-derived poliomyelitis 12 years after infection in Minnesota. New England Journal of Medicine 2011; 364: 2316-2323.

30. Modell V, et al. Global overview of primary immunodeficiencies: a report from Jeffrey Modell Centers worldwide focused on diagnosis, treatment, and discovery. Immunology 2014; 60: 132-144.

31. Hennessey KA, et al. Poliovirus vaccine shedding among persons with HIV in Abidjan, Cote d'Ivoire. Journal of Infectious Diseases 2005; 192: 2124-2128.

32. Khetsuriani $\mathbf{N}$, et al. Limited duration of vaccine poliovirus and other enterovirus excretion among human immunodeficiency virus infected children in Kenya. BMC Infectious Diseases 2009; 9: 136.

33. Duintjer Tebbens RJ, et al. Characterizing poliovirus transmission and evolution: insights from modeling experiences with wild and vaccine-related polioviruses. Risk Analysis 2013; 23: 703-749.

34. Duintjer Tebbens RJ, et al. Oral poliovirus vaccine evolution and insights relevant to modeling the risks of circulating vaccine-derived polioviruses (cVDPVs) [Review]. Risk Analysis 2013; 23: 680-702.

35. Thompson KM, Duintjer Tebbens RJ. National choices related to inactivated poliovirus vaccine, innovation, and the end game of global polio eradication. Expert Review of Vaccines 2014; 13: 221-234.

36. Dowdle WR, et al. Will containment of wild poliovirus in laboratories and inactivated poliovirus vaccine production sites be effective for global certification? Bulletin of the World Health Organization 2004; 82: 59-62.

37. Dowdle WR, et al. Containment of polioviruses after eradication: characterizing risk to improve management. Risk Analysis 2006; 26: 1449-1469.

38. Duintjer Tebbens RJ, Thompson KM. The potential benefits of a new poliovirus vaccine for long-term poliovirus risk management. Future Microbiology (in press).

39. Kew OM, et al. Vaccine-derived polioviruses and the endgame strategy for global polio eradication. Annual Review of Microbiology 2005; 59: 587-635.

40. Li L, et al. Poliovirus excretion among persons with primary immune deficiency disorders: Summary of a sevencountry study series. Journal of Infectious Diseases 2014; 210: S368-S372.

41. Modell V, et al. Global study of primary immunodeficiency diseases (PI) - diagnosis, treatment, and economic impact: an updated report from the Jeffrey Modell Foundation. Immunologic Research 2011; 51: 61-70.

42. Chapel H, et al. Common variable immunodeficiency disorders: division into distinct clinical phenotypes. Blood 2008; 112: 277-286.

43. Bousfiha AA, et al. Primary immunodeficiency diseases worldwide: more common than generally thought. Journal of Clinical Immunology 2013; 33: 1-7.

44. Boyle JM, Buckley RH. Population prevalence of diagnosed primary immunodeficiency diseases in the United States. Journal of Clinical Immonology 2007; 27: 497-502. 